\title{
Biologists join the dots
}

\author{
Tiny specks of semiconductor can make biological molecules and cellular \\ components glow in a kaleidoscope of colours. These 'quantum dots' may \\ soon be a standard biological tool, says Erica Klarreich.
}

$T$ ake some crystals of cadmium selenide, measuring a just few billionths of a metre across, add an ordinary microscope, and what have you got? According to a small but growing band of enthusiasts, this simple recipe could bring about a quantum leap in cellular imaging and biosensing.

Nanocrystals of semiconducting materials, otherwise known as quantum dots, have fascinated physicists, chemists and electronic engineers since the 1970s. The dots can confine electrons in a tiny three-dimensional space. Excite these electrons with a beam of light and they re-emit light of a precisely defined wavelength as they drop back to lower energy levels. The dots' small size gives them unusual properties that have sent researchers scurrying to investigate potential applications in electronic and optical devices - and even in a future generation of quantum computers.

Yet quantum dots are now poised to find their first practical use in the biology lab potentially doing for cell biology and other disciplines what Technicolor did for Hollywood. When excited by a pulse of light, quantum dots can be made to glow in a variety of distinct colours so intense that a relatively unsophisticated optical microscope can spot a single dot. If attached to molecules that home in on specific biological structures, quantum dots could shed light - in a rainbow assortment of hues - on the inner workings of cells.

The promise does not end there quantum dots could be used to screen candidate drugs or to provide a rapid readout of the proteins present in a particular cell or tissue. "They've got great potential," says Carolyn Larabell, a cell biologist at the University of California, San Francisco, who has been studying how cells take up the dots.

"We're just at the start of finding out what secrets they can reveal."

Although researchers are still perfecting the dots' surface chemistry, quantum dots could find their way into cell biologists' toolboxes within a year or two. Two companies - Quantum Dot Corporation in Hayward, California, and BioCrystal in Westerville, Ohio - are already preparing to market them for biological applications.

One of the main attractions of quantum

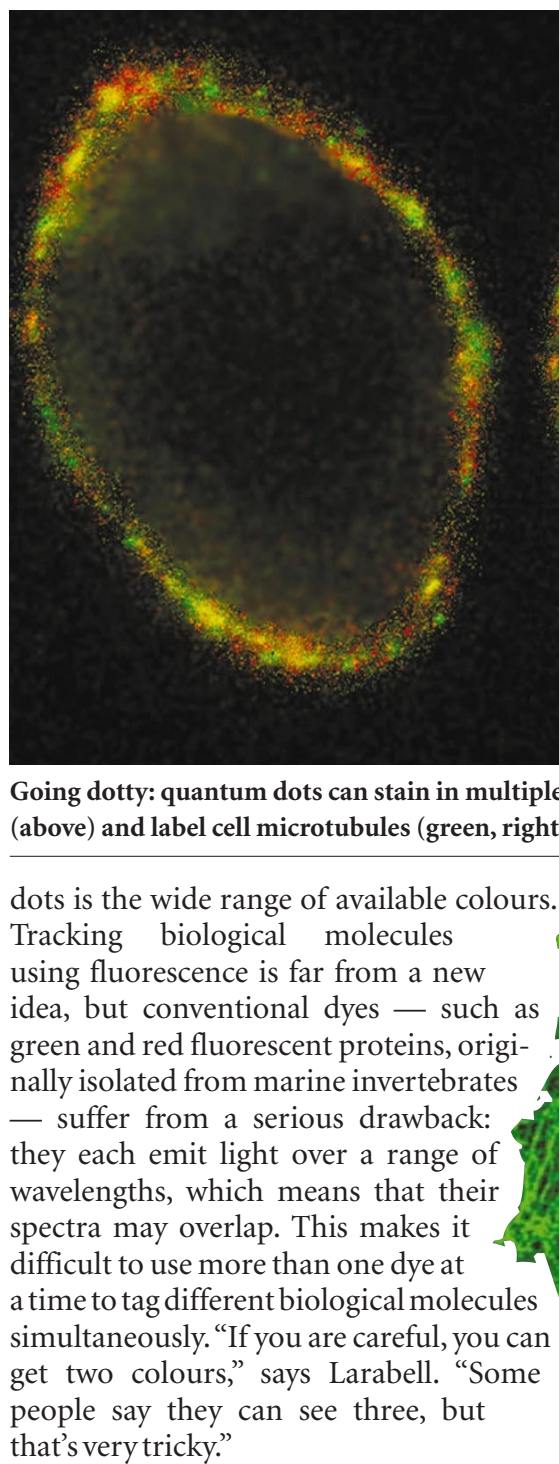

\section{Whatever hue want}

Quantum dots, on the other hand, emit light at a variety of precise wavelengths, the exact colour depending on a dot's size (see 'Pick a colour', opposite). Shuming $\mathrm{Nie}$, a quantum-dot chemist at Indiana University in Bloomington, for example, has produced dots that fluoresce in more than a dozen different colours. In principle, experts agree, it should be possible to make even more.

Another disadvantage of conventional dyes is that they can only be activated by light 
minutes, and fade rapidly if they are continually excited. The longevity of quantum dots offers the possibility of making movies of long-term interactions of biological molecules in a living cell, each biomolecule being tagged with a different colour.

This could transform the way in which cell biologists design their experiments, says Paul Alivisatos, a chemist at the University of California, Berkeley, and one of the founders of Quantum Dot Corporation. "Biologists have always had to figure out what they could do in the first 5 or 10 minutes before the dye ran out," he says. "Now they can look for much longer."

Nanocrystals of cadmium selenide can be made by mixing dimethyl cadmium and selenium in a high-temperature surfactant solution. The surfactant molecules prevent the growing crystals from merging. Once the dots have reached the desired size sometimes containing just a few dozen atoms - the solution is cooled, making the surfactant molecules coat their surfaces more effectively and so halting their growth.

Rather than using this technique, those interested in the fundamental physics and chemistry of quantum dots, as well as engineers trying to integrate them into electronic devices, instead generally produce them as part of a solid structure, in which the nanocrystals are sandwiched between layers of a different material. Assembling these structures into electronic devices poses a tough challenge, which may explain why biological applications currently lead the way. "It's very difficult to build transistors and complex circuits out of quantum dots," says Shimon Weiss of the University of California, Los Angeles, and a founder of Quantum Dot Corporation. "For biology you don't have to link them together."

For the dots to be useful for biological imaging, however, they have to be coated with substances that will make them home in on specific biomolecules. In 1998, two research groups set the ball rolling by revealing how to link quantum dots to molecules that guide them to important cellular structures.

One team, led by Alivisatos and Weiss, figured out how to attach red dots to a protein called biotin which, after forming a chain of interacting molecules, then labelled filaments of actin protein in mouse fibroblast cells. The group also linked green dots to urea and acetate groups, which carried the dots into cell nuclei, directly 'staining' them ${ }^{1}$. In independent work, a team led by Nie linked dots to antibodies and then used them to label specific target proteins in human cancer cells ${ }^{2}$.

A wide range of molecules could serve as useful guides for quantum dots. These include nucleic acids; lipids that attach to cell membranes; proteins with affinities for certain receptor proteins or sugars; and drugs,

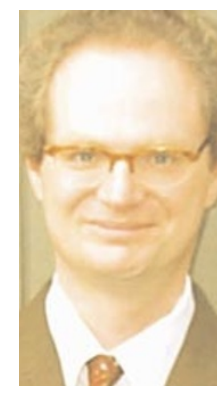

Paul Alivisatos believes the dots will transform cell biology.

\section{the behaviour of the} transport protein that pulls the neurotransmitter back into a cell after it has transmitted a signal across the gap between adjacent nerve cells.

Similar dots might also prove to be an efficient tool for screening candidate drugs, says chemist Sandra Rosenthal, a member of the team. Often, a successful drug must be able to bind to several different molecular targets to achieve the desired effect, and steer clear of other targets to avoid side-effects. Attaching different-coloured quantum dots to the various targets could make testing a simple matter, Rosenthal says. "A good hit might be a drug that displaces, say, blue, aqua and green nanocrystals where you want it to attach, but doesn't displace red, yellow and orange ones at proteins that indicate side-effects."

Quantum dots may even find a niche in medical imaging — although this will require dots that glow in the infrared spectrum. "Tissue is more or less transparent to infrared light," explains Marcel Bruchez, a senior scientist with Quantum Dot Corporation. "It can penetrate to centimetres, where with normal light it's millimetres at the most."

\section{Plugging the gaps}

This means looking beyond the current material of choice, cadmium selenide, which only emits light in the visible spectrum, and embracing other semiconductors, such as indium arsenide. Less is known about how to coat crystals of indium arsenide with molecules that will make them home in on particular biomolecules, warns Alan Waggoner, a specialist in fluorescence imaging at Carnegie Mellon University in Pittsburgh. But he is now collaborating with Quantum Dot Corporation to try to solve the problem. "It's just chemistry - you keep plugging away and sooner or later you get an answer," he says.

If the dots are to be used in humans, Waggoner adds, researchers will also have to figure out how to get our bodies to eliminate them. "You would want these things to be excreted eventually," he says.

Nie, meanwhile, is packing different combinations of quantum dots of various colours into latex beads a millionth of a metre in diameter, in carefully controlled ratios. His goal is to create a system of 'spectral fingerprints' that will allow the detection of specific genetic sequences or proteins in high-throughput biological assays. Using

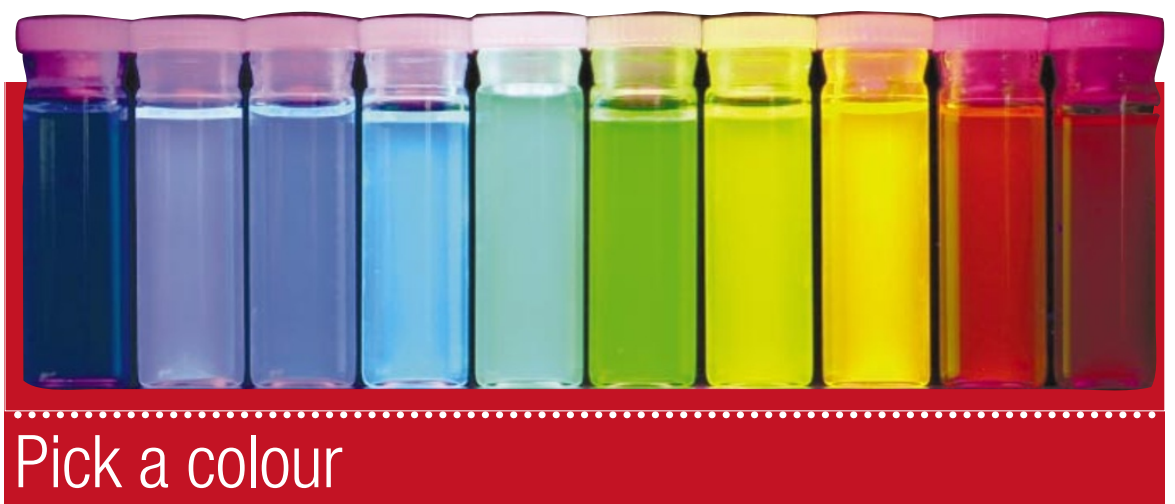

Quantum dots are nanometre-scale semiconductor crystals. When semiconductors are hit by a beam of light, some of their electrons are excited into higher energy states. As the electrons fall back to the ground state, they emit photons of light in a colour that is characteristic of the material.

When the electrons in a semiconductor are excited, they prefer to dwell at a certain fixed distance from the positive charges they leave behind, called the exciton radius. For the semiconductors used to make fluorescent quantum dots, this radius is around 5-10 nanometres.

If the entire crystal's size is less than the exciton radius, however, an effect called quantum confinement comes into play, and shifts the colour of the emitted light towards shorter wavelengths. For crystals of this size, more energy than normal is required to force electrons out of the ground state. So when the electrons return to the ground state, the photons they release have more energy — and therefore a shorter wavelength than normal.

There is a simple linear relationship between crystal size and colour: the smaller the size, the shorter the wavelength. For cadmium selenide - one of the most popular materials for making fluorescent quantum dots - varying the size of the crystals over a range of 2-6 nanometres covers the entire visible spectrum. 
dots emitting three distinct colours at ten different intensity levels, Nie already has created hundreds of beads with distinct fingerprints ${ }^{3}$. With six or seven colours, it should be feasible to extend the number of spectral fingerprints to 10,000 or more.

These beads could then be tagged with short genetic sequences to make them bind to specific DNA sequences or to the messenger RNA (mRNA) produced by active genes. The beads could also be tagged with antibodies so that they recognize specific proteins. By mixing a liquidized biological sample with a solution of beads tagged in this way, the beads could be used to provide a rapid read-out of the genes that are active, or the proteins that are present, in the sample.

The idea would be to label all of the mRNA or protein in a sample with a conventional dye, or a quantum dot of a particular colour. After the sample is mixed with the quantum-dot-labelled beads, the beads would be fed through a flow cytometer, a device that funnels them one-by-one across a small opening. Here, a laser-based system would read the beads' spectral fingerprints and identify the beads that had bound to a target molecule.

Currently, high-throughput analyses of gene expression are conducted using 'chips' or microarrays, glass slides on which hundreds or thousands of DNA sequences are immobilized. Biological samples in which all the mRNA sequences have been

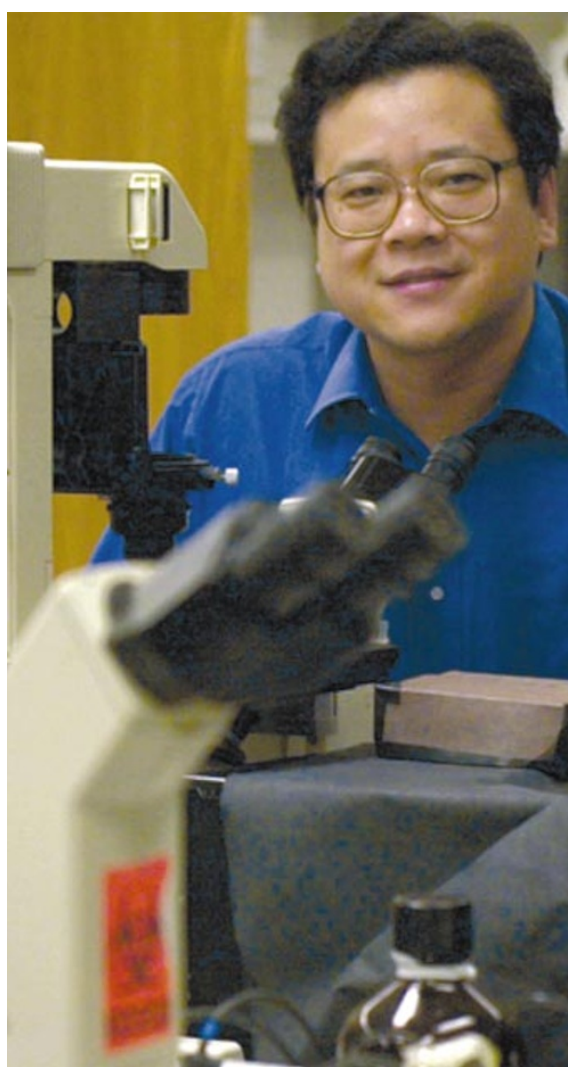

Screen saver: Shuming Nie says the dots could solve the problem of screening for many proteins.

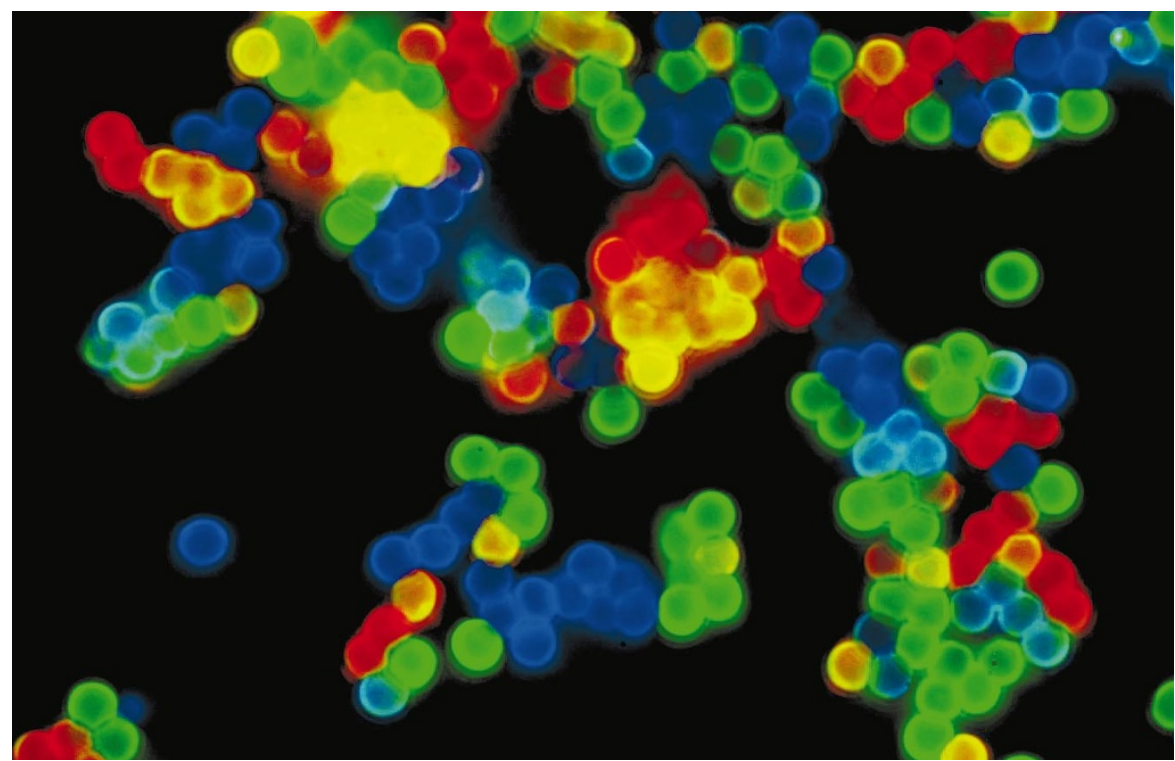

Dot detectives: latex beads with 'spectral fingerprints' of dots could be used to detect gene expression.

fluorescently tagged are washed over the chip, and lasers are used to determine where on the chip the mRNAs bind - showing which genes were active in the sample. Nie argues that his approach should provide results more quickly. "DNA chips require about 24 hours," he says. "The beads would just take 10-15 minutes."

\section{Flexible friends}

Quantum-dot beads would also be more flexible. In the microarray approach, adding new genes to the analysis means producing a new chip. But analyses in solution have no such limitations. "You just dump in the beads, and you can add new ones or remove old ones as you like," says Alivisatos. "It just might make the difference between a few people being able to do these assays at great expense, and people being able to do them very widely."

In the long run, says Nie, the main benefit could be in the emerging field of proteomics, as it is proving difficult to design 'protein chips' to screen for the presence of proteins in the way that DNA microarrays screen for gene activity. Quantum-dot beads tagged with antibodies might provide the answer, Nie suggests. Unlike flat chips, beads in solution could allow antibodies to maintain their normal three-dimensional structure, which is crucial to their functioning.

But it is in the field of cell biology that quantum dots may have their biggest impact. For biologists who want to monitor the interactions between biological molecules or cellular components over long periods of time, says Alivisatos, quantum dots could "change their whole mode of operation".

For instance, it may become possible to determine in living cells whether two proteins are linked together into a complex. When proteins tagged with dots are phys- ically linked in this way, the dots are close enough together that light emitted from one dot can activate the other, making it glow more brightly than normal. "This could give us information about the dynamic interaction of two proteins," says Randy Blakely, a neuroscientist at Vanderbilt University who works with Rosenthal. "The optical resolution of a normal fluorescent microscope isn't enough to tell whether molecules are physically interacting with each other."

Some technical problems remain to be ironed out, however, mostly relating to the dots' surface chemistry. "We need to protect the quantum dots so that they are not oxidized, are very stable, can withstand salt concentrations in cells, and don't bind to what you don't want them to," says Weiss. The very versatility of quantum dots - the fact that their cellular target can be changed simply by changing their surface chemistry - is both a blessing and a curse, Bruchez adds, as it means that the surface must be tailored for each biological application. "We're looking for a robust surface that is widely applicable," he says.

Although neither BioCrystal nor Quantum Dot Corporation has figured out yet how to mass-produce the dots cheaply, both have started making their dots available to small groups of researchers, and plan to expand their distribution in the coming year.

Once the dots become more widely available, Alivisatos says, it might take biologists a while to explore their potential. But Larabell is confident that this won't take long. "When the methods are worked out, they'll be used instantly," she predicts.

Erica Klarreich is an intern with Nature.

\footnotetext{
1. Bruchez, M. Jr, Moronne, M., Gin, P., Weiss, S. \& Alivisatos, A. P. Science 281, 2013-2016 (1998).

2. Chan, W. \& Nie, S. Science 281, 2016-2018 (1998).

3. Han, M., Gao, X., Su, J. \& Nie, S. Nature Biotechnol. 19, 631-635 (2001).
} 